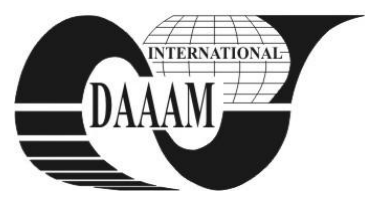

\title{
TESTING THE EFFECT OF PERMANENT MAGNETS ON MAGNETIC NANOPARTICLES FERROFLUID - TARGETED DELIVERY INSIDE KNEE ARTICULATION
}

\author{
LAPTOIU, D[an] C[onstantin]; ANTONIAC, I[ulian] \& ANTONIAC, A[urora]
}

\begin{abstract}
The paper presents the development of a medical device - knee orthosis for joint articular disease therapy using magnetic nanomaterials. The main stages of the research regarding magnetic nanoparticles synthesis and coating, magnetic field effects on the magnetic ferrofluid, as well as orthosis design criteria and optimal magnets configuration on the brace are described.
\end{abstract}

Key words: permanent magnets, magnetic nanoparticles, magnetic field, ferrofluid

\section{INTRODUCTION}

Various solutions (Marshall, 2003), (Juni, 2007), (Kon, 2011) have been proposed for pain treatment in degenerative joint damage (such as osteoarthritis) in order to minimize the pain, maintain or improve joint mobility and minimize functional deterioration. One of these solutions is magnetically controlled viscosupplimentation therapy which aims to restore the viscoelastic qualities of the joint fluid, with concomitant improvement of rheology as a result of controlled degradation of hyaluronate into the joint. This therapy requires the use of a special brace (orthosis) modified to allow insertion of groups of permanent magnets. The magnetic field thus created potentially localizes and concentrates the magnetic ferrofluid with surface covered nanoparticles, enhancing the targeted delivery of active substances as hyaluronates.

Preliminary research led to the definition of potential mechanisms of action of the method: 1. restoration of viscoelasticity: the joint has a more elastic manner to react to stress, depending on the workload; 2. anti-inflammatory effect due to hyaluronate and cortisone derivatives coatings associated with the nanoparticles

3. analgesic (pain relief) effect (with the use of medical magnets);

4. joint protection: clinical studies with arthroscopic control after using different specialised orthoses, suggests a slower rate of progression of osteoarthritis - protective effect described by (Hewett, 1998).

This complex system designed with the therapeutic method has the following advantages: adaptability - the device can be used in multiple joint areas, the therapy is targeted to the affected area, simple handling during treatment, avoid joint stiffness allowing kinetotherapy.

\section{MAGNETIC NANOPARTICLES RETENTION TESTING}

Magnetic nanoparticles were prepared using coprecipitation technique from $\mathrm{FeCl}_{2}$ and $\mathrm{FeCl} 3$ in a concentrated aqueous alkaline solution $\left(\mathrm{NH}_{4} \mathrm{OH} 25 \%\right)$ as a mixture of iron salts in molar ratio $\mathrm{FeCl}$ : $\mathrm{FeCl} 3$ of 1: 2,7. The magnetic nanoparticles were coated in a layer by layer method using tetramethylammonium hydroxide (TMOH). Tests were performed for 4 solutions with different magnetic nanoparticles concentrations for assessing the effect of a constant $1.2 \mathrm{~T}$ (12300 Gauss) magnetic field (created by a neodymium iron boron - NdFeB magnet with dimension of 20x10x5 mm) on nanoparticles retention on the recipient walls. After initial calculations of the flux density of a plain rectangular magnet at various distances, N35 and N40 were selected for further testing. Two parameters were modified during experiments: time exposure (3, 10 and 15 minutes) and the distance between the magnet and recipient $(3,5$ and $12 \mathrm{~mm})$.

Figure 1 presents the formula used to calculate the flux density at given incremental distances $X$ above the surface and on the centre-line of a rectangular shaped magnet. Best results were provided by N38, N40 permanent magnets.

Figure 2 shows a simulation of the magnetic field produced by $4 \times 4$ magnets arrangement in order to potentially target the femuro-patellar area of the knee joint (COMSOL software for magnetic field analysis).

Figure 3 presents one of the experimental tests performed for analyzing the effects of magnetic field on the different sample.

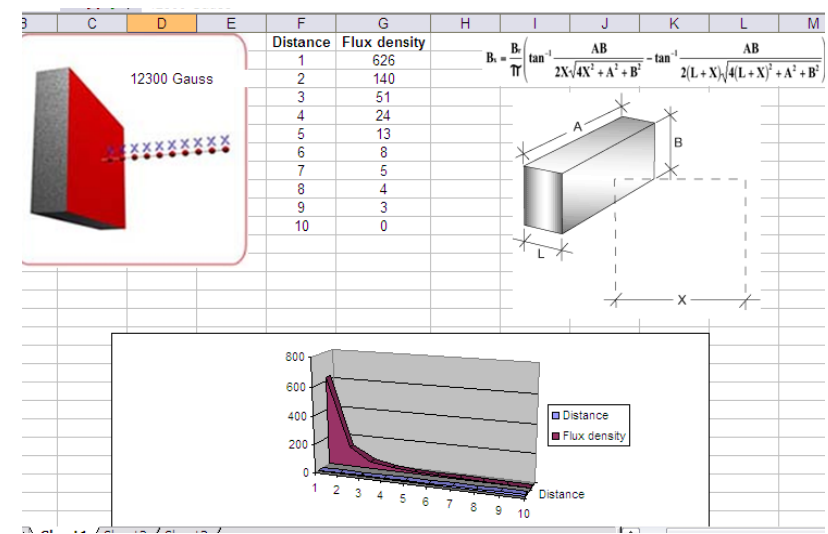

Fig. 1. Magnetic flux density calculations

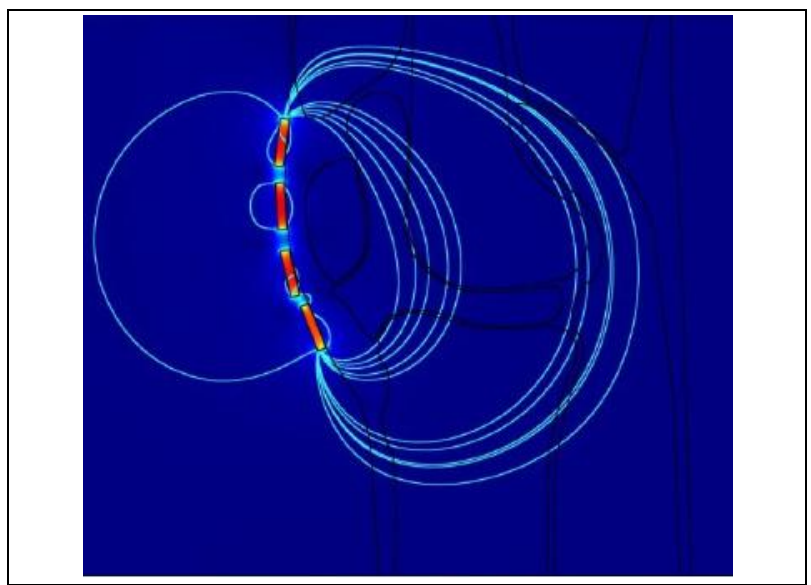

Fig. 2. Experimental simulation of the $4 \mathrm{x} 4$ permanent magnet matrix 


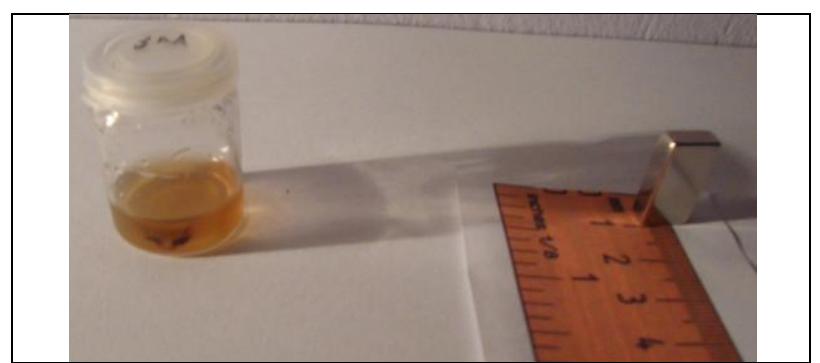

Fig. 3. Experimental tests for analyzing the effects of magnetic field

Samples meet concentrations of $3 \mathrm{M} 1 \%, 10 \% 1 \mathrm{M}$ and $8 \mathrm{M}$ $100 \%$. 3M solution contained in the container is covered as constituted by the magnetic nanoparticles of magnetite and polymer coating. In the figure 3 we see that experimental tests to analyze the effects of magnetic field on the $1 \mathrm{M}$ sample which exhibited the highest magnetic retention, initially using a single magnet and then a $2 \times 2$ matrix and $4 \times 4$ magnets. As we increase the distance between samples and magnets, the attracted particle number is reduced. After three minutes we can already see how the magnet $1 \mathrm{M}$ and $3 \mathrm{M}$ samples have accumulated a number of magnetic particles on the wall. The tests showed that only a few particles moved by the wall, the displacement being hampered by the viscous hyaluronic acid composition. After an exposure of 15 minutes, a $40 \%$ of aggregate remained near the magnet wall for $3 \mathrm{M}$ sample. Keeping the two components of the device for 45 minutes will provide the best magnetic retention. The $1 \mathrm{M}$ sample, having a higher concentration of particles, requires a long interaction with the magnet in order to have higher retention. $8 \mathrm{M}$ sample shows no visible change, because of too high concentration of magnetic particles and coatings.

\section{DESIGN CRITERIA FOR A MAGNETIC KNEE ORTHOSIS}

Analysis of the orthoses market shows the lack of standardization in the field, in spite of the existence of common design elements such as adjustable rods, metallic hinges or adjustable Velcro straps. Moreover, despite a growing number of new orthoses models, a clinical comparative evaluation of these devices short and long term effect on the knee rehabilitation has not been performed. This is also the case of magnetic knee braces, which are currently used by patient mostly for pain relief, even if systematic studies regarding the influence of different magnets configurations on the knee joint fluid are not found in the literature. Therefore, aside of general design criteria for knee braces (simplicity, light weight, stability, ability to limit knee motion without affecting knee function, comfort, etc., as they are presented in literature (Ragalbuto, 1989), (Cherry, 2006), (Singer\&Lamontagne, 2008)), the magnetic knee orthosis designed in the ARTROMAG project focused also on the optimal magnets configuration on the orthosis using Comsol Multiphysics software in two knee positions $\left(0^{\circ}\right.$ and $\left.90^{\circ}\right)$.

Figure 4 presents the design of the knee orthosis with the permanent magnets matrix in place in order to target the femuro-patellar area.

\section{CONCLUSIONS}

In conclusion, the objective of having a magnetic retention is best achieved with N40 permanent magnets for a period of at least 15 minutes. Insertion of the device in a tissue with similar characteristics the knee joint application, caused a significant obstacle that attenuates the effect of magnetic field. This will generate the need to increase the power of attraction of the magnet to get results (minimum $4 \times 4$ arrangements).

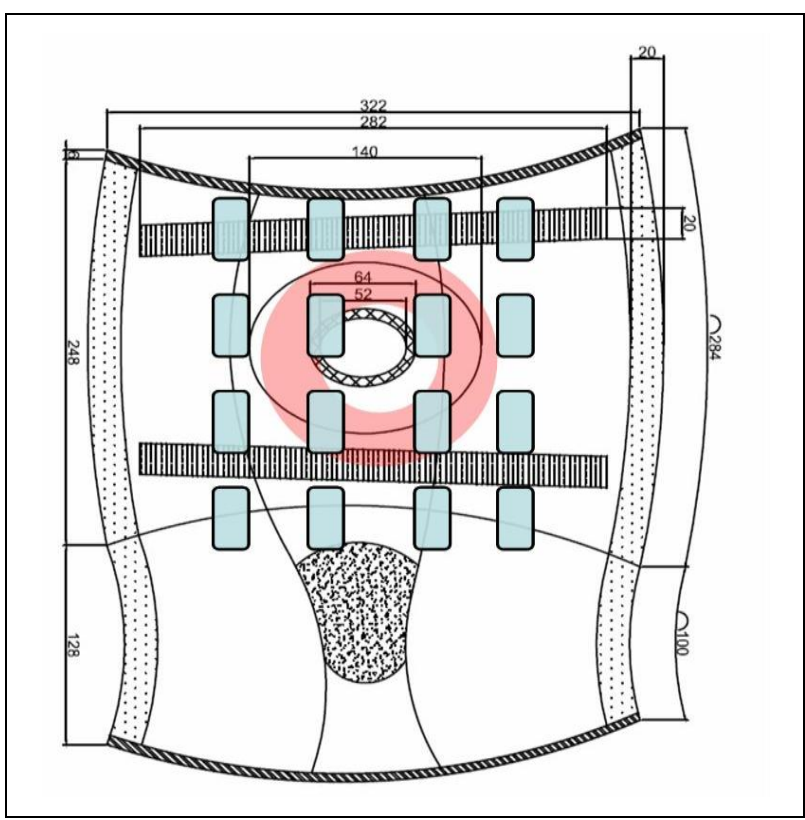

Fig. 4. Design of the knee orthosis with the permanent magnets

\section{ACKNOWLEDGEMENTS}

This work was supported by PNCDI2 Project 42-132, ARTROMAG - Medical device for joint articular disease therapy using nanomaterials and magnetic field effects.

\section{REFERENCES}

Marshall, K.W. (2003). Intra-articular hyaluronan therapy. Foot Ankle Clin, 8:221-232.

Jüni, P., et al. (2007). Efficacy and safety of intraarticular hylan or hyaluronic acids for osteoarthritis of the knee: A randomized controlled trial. Arthritis Rheum., 56, pp. 36103619

Gijs, M.A.M. (2010). Microfluidic applications of magnetic particles for biological analysis and catalysis, Chem. Rev. 110, pp.1518-1563

Self, B.P., et al. (2000). Biomechanical Analysis of a Medial Unloading Brace for Osteoarthritis in the Knee. Arthritis Care Research, 13, 4, pp.191-197

Draper, E.R.C., et al. (2000). Improvement in function after valgus bracing of the knee. The Journal of Bone and Joint Surgery, 82-B/7, pp.1001-1005

Giori, N.J. (2004). Load-shifting brace treatment for osteoarthritis of the knee: A minimum 21/2-year follow-up study. Journal of rehabilitation Research \& Development, 41/2, pp.187-194

Nadaud, M.C., et. al. (2005). In Vivo Three-Dimensional Determination of OA Brace Effectiveness: A Multiple Brace Analysis, American Academy of Orthopaedic Surgeons 72nd Annual Meeting,

Ragalbuto, M.A., et al. (1989). The forces in a knee brace as a function of hinge design and placement, Am J Sports Med July, 17, pp.535-543

Cherry, M.S., et al. (2006). Design and fabrication of an elastic knee orthosis-Preliminary results, Proceedings of IDETC/CIE 2006, ASME 2006 International Design Engineering Technical Conferences \& Computers and Information in Engineering Conference, September 10-13, Philadelphia, USA, pp.3-9

Singer, J.C., Lamontagne, M. (2008). The effect of functional knee brace design and hinge misalignment on lower limb joint mechanics, Clinical Biomechanics, 23, pp.52-59 